\title{
Quetiapine-Induced Hypomania and its Association with Quetiapine/Norquetiapine Plasma Concentrations: A Case Series of Bipolar Type 2 Patients
}

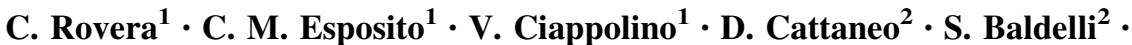 \\ E. Clementi ${ }^{3,4}$ - A. C. Altamura ${ }^{1} \cdot$ M. Buoli $^{1}$
}

Published online: 23 October 2017

(c) The Author(s) 2017. This article is an open access publication

\begin{abstract}
International guidelines consider quetiapine at medium doses (300-400 mg/day) as valid options for the treatment of bipolar depression for the supposed lower risk of a switch to hypomania/mania than antidepressants. Norquetiapine is an active metabolite with antidepressant action. We describe three cases of induced hypomania in bipolar type 2 subjects who received quetiapine extendedrelease monotherapy $(300 \mathrm{mg} / \mathrm{day})$ for a mild/moderate major depressive episode. Quetiapine and norquetiapine plasma concentrations were measured after 1 week of treatment. Hypomania appeared after 7-10 days of quetiapine extended-release monotherapy and all subjects had a quetiapine/norquetiapine plasma concentration ratio $<1$. We propose a ratio value $<1$ as a predictor of risk for a switch to hypomania in bipolar depressed subjects receiving quetiapine extended-release monotherapy. Future research should ascertain the validity of this laboratory parameter to assess the risk of quetiapine-induced hypomania in large samples of bipolar patients.
\end{abstract}

Keywords Bipolar depression · Hypomania - Quetiapine

M. Buoli

massimiliano.buoli@hotmail.it;

massimiliano.buoli@unimi.it

1 Department of Psychiatry, University of Milan, Fondazione IRCCS Ca'Granda Ospedale Maggiore Policlinico, Via F. Sforza 35, 20122 Milan, Italy

2 Clinical Pharmacology Unit, ASST Fatebenefratelli-Sacco, Milan, Italy

3 Clinical Pharmacology Unit, Consiglio Nazionale delle Ricerche, Institute of Neuroscience, Department of Biomedical and Clinical Sciences, L. Sacco University Hospital, Università degli Studi di Milano, Milan, Italy

4 E. Medea Scientific Institute, Bosisio Parini, Italy

\section{Key Points}

Quetiapine monotherapy is a therapeutic option for the treatment of depressive symptoms in bipolar type 2 patients.

Quetiapine has a potential risk of induced hypomania owing to the antidepressant effect of its metabolite norquetiapine.

A ratio of quetiapine/norquetiapine plasma concentrations $<1$ is thought to be a risk factor for developing quetiapine-induced hypomania.

\section{Introduction}

Bipolar disorder (BD) is a prevalent psychiatric condition (affecting up to 5-6\% of the general population) [1] associated with high disability and a risk of chronicity if not promptly treated [2]. Approved in 1997 by the US Food and Drug Administration for the treatment of acute episodes of schizophrenia, quetiapine has been shown to be effective in a large number of neuropsychiatric disorders, including BD [3, 4]. Currently, both the European Medicines Agency and the US Food and Drug Administration recommend quetiapine for the treatment of major depressive and manic episodes as well as for the prevention of recurrences in BD. With regard to acute mood episodes, the World Federation of Societies of Biological Psychiatry guidelines consider quetiapine as a compound with high evidence of efficacy at the dosage of $600-800 \mathrm{mg} / \mathrm{day}$ for 
acute mania [5] and at the dosage of 300-600 mg/day for bipolar depression [6]. In addition, according to the Canadian Network for Mood and Anxiety Treatments [7], quetiapine is the only drug recommended as first-line treatment for major depressive episodes in BD type 2 .

Quetiapine appears to be one of the best options in terms of efficacy in bipolar depression and of risk of switching to hypomania/mania [8]. Quetiapine-induced hypomania or mania has been previously described [9, 10]; however, because the cases of quetiapine-induced hypomania are extremely rare in comparison with the antidepressant-induced cases $[11,12]$, quetiapine remains widely prescribed in the clinical practice for the treatment of bipolar depression [13].

Norquetiapine, the most important active metabolite of quetiapine, has been shown to be associated with antidepressant, anxiolytic, and neurotrophic effects in humans [14]. In particular, norquetiapine facilitates dopamine release in the prefrontal cortex and inhibits dopamine reuptake $[15,16]$. Moreover, norquetiapine potentiates serotoninergic and noradrenergic transmission through its high affinity for $5-\mathrm{HT}_{1 \mathrm{~A}}, 5-\mathrm{HT}_{2 \mathrm{C}}$, and $5-\mathrm{HT}_{7}$ receptors and through noradrenaline reuptake inhibition [17, 18]. Previous studies have showed that the ratio of norquetiapine/quetiapine plasma concentrations was associated with an improvement of mood symptoms in bipolar patients $[19,20]$. In the first study [19], bipolar patients showed a curvilinear relationship between the norquetiapine/quetiapine concentration ratio and the improvement of depressive symptoms after 3 months of treatment with quetiapine. In the second and more recent study [20], bipolar patients were found to have a significant correlation between improvement of depressive symptoms and the norquetiapine/quetiapine ratio, measured after 3 months of quetiapine treatment. In other studies, however, norquetiapine has been reported as possibly responsible for a switch to hypomania in bipolar subjects in treatment with quetiapine $[9,10]$, thus the measurement of norquetiapine plasma concentrations might be useful to evaluate the risk of a switch to hypomania in depressed bipolar subjects. In this article, we describe three cases of quetiapine-induced hypomania in subjects who were monitored for quetiapine and norquetiapine plasma concentrations.

\section{Case Series}

Three bipolar type 2 patients with a moderate major depressive episode according to the Diagnostic and Statistical Manual of Mental Disorders, Fifth Revision [21] had a switch to hypomania during quetiapine monotherapy. They were outpatients of the Department of Psychiatry, University of Milan. The patients provided consent to share their clinical data for research purposes. All patients underwent laboratory evaluations such as blood cell count, renal function tests, pancreatic and liver enzymes, and also safety assessments (neurological examination and electrocardiography). All the examination results were normal. All subjects were treated with $300 \mathrm{mg} /$ day of quetiapine extended release (ER). After 1 week of treatment, quetiapine and norquetiapine plasma concentrations were measured, considering that steady state is usually achieved after 3 days of sequential doses [22]. Young Mania Rating Scale (YMRS), Hamilton Depression Rating Scale (HAM-D), Hamilton Anxiety Rating Scale (HAM-A), and Brief Psychiatric Rating Scale (BPRS) were administered after 1 week (T1) and after 1 month of treatment (T2). Hypomania was defined not only according to the Diagnostic and Statistical Manual of Mental Disorders, Fifth Revision criteria, but also by an YMRS score $\geq 10$ [23]. The patients did not receive other psychotropic drugs during the follow-up period. In addition, all the patients had discontinued autonomously the previous pharmacotherapy for at least 3 months. None of the subjects developed side effects except for induced hypomania. Changes in rating scale scores for the three patients are reported in Fig. 1.

The first case was a 28-year-old woman who had the first diagnosis of BD type 2 in 2007, while experiencing a hypomanic episode subsequent to a major depressive episode. In the last years, she experienced two further major depressive episodes and three hypomanic episodes. She has a family history of psychiatric disorders: her mother experienced a major depressive disorder, while one sister and one brother received a diagnosis of generalized anxiety disorder. Substance misuse was never reported by the patient, while she experienced Hashimoto thyroiditis (currently in remission and treated with hormone replacement therapy) and polycystic ovary syndrome (currently in remission and treated with a combined hormonal contraceptive). At the time of the first evaluation (T0), she presented with a mild major depressive episode (YMRS = 8; HAM-D $=17$; HAM-A $=28 ; \quad$ BPRS = 38). Pharmacotherapy with quetiapine ER $300 \mathrm{mg}$ /day was prescribed and, after 1 week (T1), quetiapine and norquetiapine plasma concentrations were 113.9 and $178.1 \mathrm{ng} / \mathrm{mL}$, respectively (ratio $=0.64$ ). The scores of the clinical rating scales were the following: T1: YMRS $=11$; HAM$\mathrm{D}=13 ;$ HAM-A $=25 ; \mathrm{BPRS}=36 ; \mathrm{T} 2$ : $\mathrm{YMRS}=14$; HAM-D $=8 ;$ HAM-A $=23 ; \quad \mathrm{BPRS}=32$. The clinical rating scale scores show the improvement of depressive symptoms, but also the early switch to hypomania (after 1 week of treatment).

The second case was a 45-year-old woman who first visited our outpatient clinic in 2005 because of a hypomanic episode followed by a major depressive episode. At that time, she had already presented with several mood 

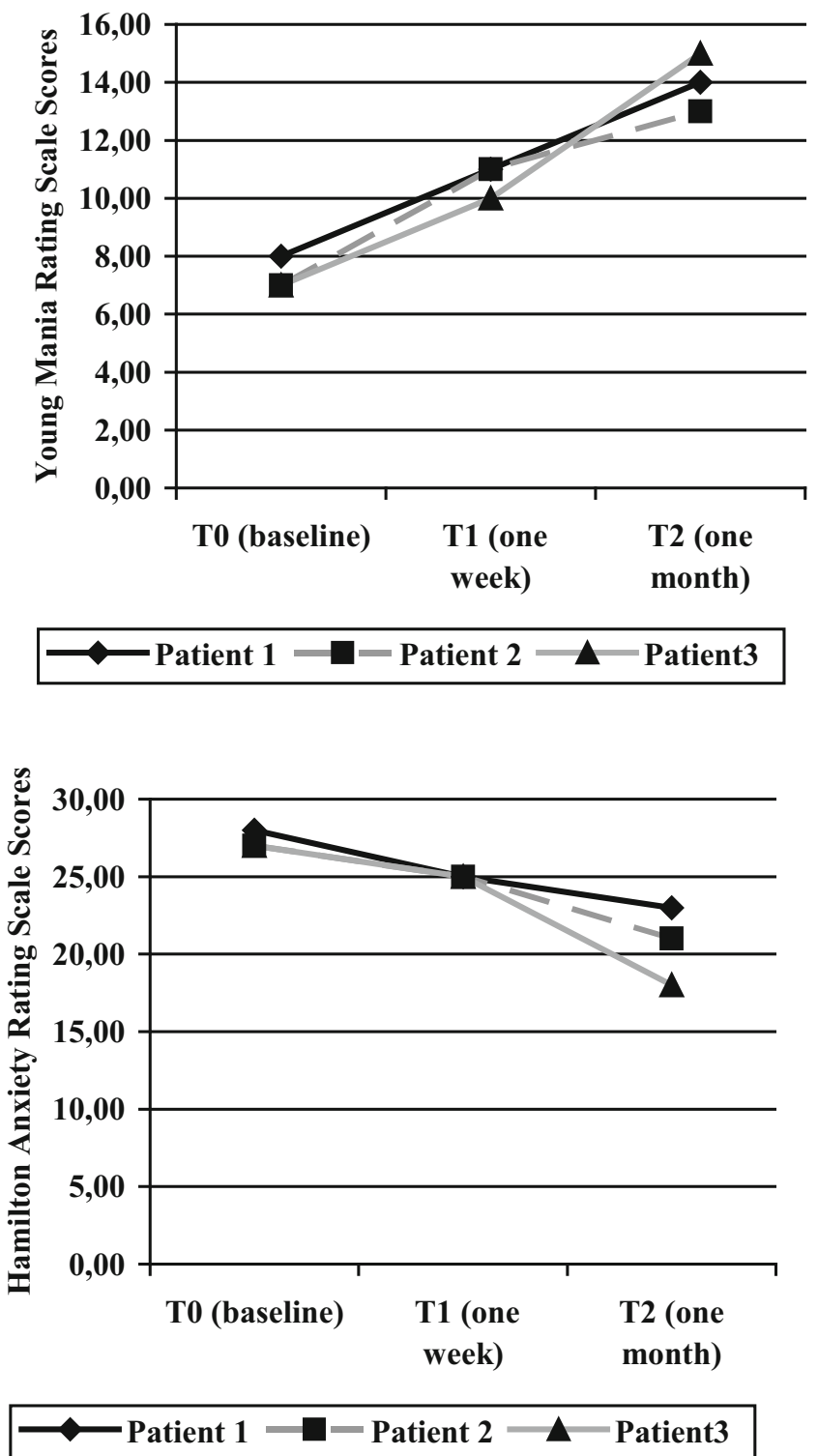
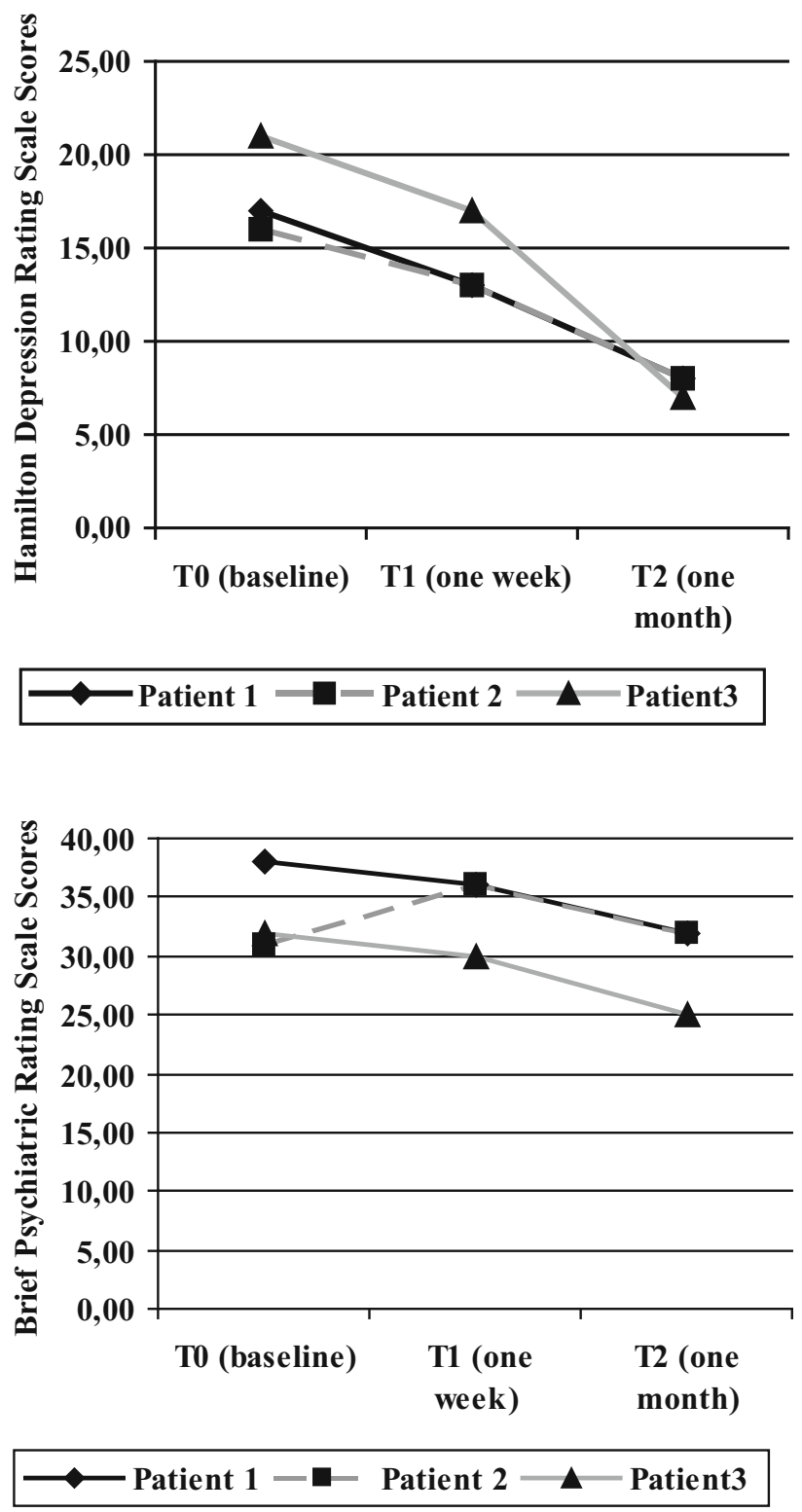

Fig. 1 Rating scale scores of the three patients during the 1-month follow-up period

episodes, starting from the age of 20 years, when she received a diagnosis of BD type 2, and she had been hospitalized three times for major depressive episodes. Her father had experienced a major depressive disorder, while her mother presented with a generalized anxiety disorder. No substance abuse was reported by the patient, while she had a head trauma following a road accident in 1998 at the age of 26 years, without any long-term consequences (no specific lesions were documented by a subsequent brain scan with computerized tomography). At baseline (T0), the patient presented with a mild major depressive episode (YMRS = 7; HAM-D = 16; HAM-A = 27; BPRS = 31) and pharmacotherapy with quetiapine ER $300 \mathrm{mg} /$ day was prescribed. After 1 week (T1), quetiapine and norquetiapine plasma concentrations were respectively 5 and $10.1 \mathrm{ng} / \mathrm{mL}$ (ratio $=0.59$ ). The rating scale scores were the following: T1: YMRS $=11$, HAM-D $=13$, HAM-A $=25, \quad$ BPRS $=36 ;$ T2: $Y M R S=13$, HAM$\mathrm{D}=8, \mathrm{HAM}-\mathrm{A}=21, \mathrm{BPRS}=32$. The patient presented with an improvement of depressive symptoms, but also an early switch to hypomania (after 1 week of treatment).

The third case was a 25-year-old woman who developed obsessive-compulsive symptoms at the age of 12 years. She has a family history of psychiatric disorders: her father and her grandmother were affected by major depressive disorder, while her brother and an aunt experienced a generalized anxiety disorder. Neither substance abuse nor medical comorbidity was reported by the patient. When she 
started university at the age of 19 years, she showed a worsening of obsessive symptoms and received treatment with citalopram $25 \mathrm{mg} /$ day for 1 year. There was an improvement of obsessive-compulsive symptoms, but also a switch to hypomania, followed by a major depressive episode. In the last 5 years, the patient presented with several episodes of mood alteration (including three spontaneous hypomanic episodes, two major depressive episodes, and one episode of antidepressant-induced hypomania) and has been followed up at our outpatient clinic with a diagnosis of BD type 2 . The patient received only citalopram during major depressive episodes (the last two treatments in combination with a mood stabilizer or atypical antipsychotic different from quetiapine). At T0, she experienced a moderate major depressive episode (T0: YMRS = 7; HAM-D = 21; HAM-A = 27; BPRS = 32) and pharmacotherapy with quetiapine ER $300 \mathrm{mg} /$ day was prescribed. The patient showed an amelioration of depressive symptoms, but also a switch to hypomania (after 10 days). The rating scale scores were the following: T1: $\mathrm{YMRS}=10, \mathrm{HAM}-\mathrm{D}=17, \mathrm{HAM}-\mathrm{A}=25, \mathrm{BPRS}=30$; T2: $\quad$ YMRS $=15, \quad$ HAM-D $=7, \quad$ HAM-A $=18$, $\mathrm{BPRS}=25$. After 1 week (T1), quetiapine and norquetiapine plasma concentrations were found to be respectively, 33.7 and $42.5 \mathrm{ng} / \mathrm{mL}$ (ratio $=0.79$ ).

After the hypomanic switch, all three patients received higher doses of quetiapine, which were titrated up to $800 \mathrm{mg} /$ day to have an anti-manic effect. However, no cytochrome P450 3A4 inhibitor was introduced in the treatment of these patients. During the period between T1 and $\mathrm{T} 2$, none of the patients satisfied the criteria for a major depressive episode with mixed features. In the next month after T2, patients showed an amelioration of hypomanic symptoms till the remission (YMRS $<7$ ). No patients developed substantial depressive symptoms (HAM-D < 4). This is not surprising as a recent report showed quetiapine is a slow but effective anti-manic agent [24].

\section{Discussion}

The amelioration of depressive symptoms with quetiapine at the dosage of $300 \mathrm{mg} /$ day in the three presented cases is in line with reports in the literature indicating that quetiapine ER at this dosage has antidepressant efficacy [8]. Despite the well-known metabolic side effects, this drug appears to also be a valid option for the treatment of major depressive episodes in bipolar type 2 patients with a supposed lower risk of hypomania with respect to antidepressants [25]. The novelty of the reported cases is that quetiapine is associated with a potential risk of hypomania, a situation not previously described. We also show that clinical biological/predictors may be useful to detect susceptibility to a mood switch in bipolar subjects receiving quetiapine monotherapy. The association between quetiapine monotherapy and mood switch is demonstrated by the fact that the medical and psychiatry comorbidities of the three bipolar subjects, if present, were in remission and the patients were not treated with other compounds with the exception of the first patient who received hormone replacement therapy. In addition, the patients had no history of rapid cycling and 7-10 days is too short a period to consider a spontaneous remission of depressive symptoms [26]. Rapid cycling was excluded according to the Diagnostic and Statistical Manual of Mental Disorders, Fifth Revision criteria (four or more mood episodes/year) [27]. Finally, the patients had mostly a clinical history characterized by hypomanic episodes that started during euthymic phases and some months after the remission of depressive symptoms. Although there can be no absolute certainty, it is reasonable to exclude that hypomania may be the result of the natural course of BD. Taking into consideration all the mentioned aspects, according to the Naranjo Adverse Drug Reaction Probability Scale, hypomania as an adverse reaction of the administration of quetiapine ER (300 mg/day) may be considered as probable in the light of a score of 6 [28].

Quetiapine and norquetiapine plasma concentrations in the described bipolar subjects showed a high degree of variation, most likely owing to the inter-variability in cytochrome P450 functioning [29], as the patients did not take drugs with pharmacokinetic interactions with quetiapine or they were smokers. The patients took the therapy at 10 p.m. and compliance was checked by counting the pills in the blister. In addition, caregivers ensured treatment compliance.

As the efficacy of an antidepressant is mainly the result of the metabolite norquetiapine and there is a substantial variability of quetiapine/norquetiapine plasma concentrations after the same dose of quetiapine ER, the ratio of the plasma concentrations of quetiapine/norquetiapine may be a useful tool to predict the risk of mood destabilization [30]. Of note, a ratio $<1$ is proposed as a pharmacological predictor for a switch to hypomania in bipolar subjects receiving medium doses of quetiapine. In a previous study published by our group, bipolar patients who had ameliorated the depressive symptoms without developing hypomania showed a quetiapine/norquetiapine ratio $>1$ [20]. The results, however, are not totally comparable to those of the present case series as the evaluation of drug plasma concentration was done after 3 months of treatment and the sample consisted of both bipolar type 1 and 2 patients.

Prospective studies with large samples of bipolar patients are needed to confirm these preliminary results. In addition, clinicians should evaluate if a combined therapy (e.g., with valproate) may prevent the risk of quetiapine- 
induced hypomania and if the prophylactic efficacy overcomes the increased risk of side effects due to combined therapies [4].

\section{Conclusions}

Even though quetiapine monotherapy ( $300 \mathrm{mg} /$ day) is an option for the treatment of depressive symptoms of bipolar type 2 patients, clinicians should take into account the potential risk of induced hypomania. Some bipolar type 2 subjects may have a predisposition to a switch to hypomania when treated with low-medium doses of quetiapine monotherapy. Future studies need to assess the size of the risk and identify the risk factors of induced hypomania/mania. A ratio $<1$ of quetiapine/norquetiapine plasma concentrations might be a useful predictor of the risk of a mood switch. The validation of this laboratory parameter should be examined by future research as well as the evaluation of the efficacy of a combined therapy (e.g., quetiapine plus valproate) in preventing the risk of a mood switch in bipolar depressed subjects receiving quetiapine monotherapy.

\section{Compliance with Ethical Standards}

Funding No financial support was received for the conduct and preparation of this case series.

Conflict of interest Prof. A. Carlo Altamura has served as a consultant for Merck and Astra Zeneca and has been on the speaker bureau for Sanofi, Lilly, and Pfizer. Dr. Massimiliano Buoli has served as a consultant for Lundbeck. Prof. Emilio Clementi, Drs. Chiara Rovera, Valentina Ciappolino, Dario Cattaneo, Sara Baldelli, and Ms Cecilia Maria Esposito have no conflicts of interest directly relevant to the content of this case series.

Informed consent Written informed consent was obtained from all three patients for the publication of this case report.

Open Access This article is distributed under the terms of the Creative Commons Attribution-NonCommercial 4.0 International License (http://creativecommons.org/licenses/by-nc/4.0/), which permits any noncommercial use, distribution, and reproduction in any medium, provided you give appropriate credit to the original author(s) and the source, provide a link to the Creative Commons license, and indicate if changes were made.

\section{References}

1. Merikangas KR, Jin R, He JP, et al. Prevalence and correlates of bipolar spectrum disorder in the world mental health survey initiative. Arch Gen Psychiatry. 2011;68:241-51.

2. Altamura AC, Serati M, Buoli M. Is duration of illness really influencing outcome in major psychoses? Nord J Psychiatry. 2015;69(6):403-17.

3. Sanford M. Quetiapine extended release: adjunctive treatment in major depressive disorder. CNS Drugs. 2011;25:803-13.
4. Buoli M, Serati M, Altamura AC. Is the combination of a mood stabilizer plus an antipsychotic more effective than mono-therapies in long-term treatment of bipolar disorder? A systematic review. J Affect Disord. 2014;152-154:12-8.

5. Grunze H, Vieta E, Goodwin GM, et al. The World Federation of Societies of Biological Psychiatry (WFSBP) guidelines for the biological treatment of bipolar disorders: update 2009 on the treatment of acute mania. World J Biol Psychiatry. 2009; $10: 85-116$

6. Grunze H, Vieta E, Goodwin GM, et al. The World Federation of Societies of Biological Psychiatry (WFSBP) guidelines for the biological treatment of bipolar disorders: update 2010 on the treatment of acute bipolar depression. World J Biol Psychiatry. 2010;11(2):81-109.

7. Yatham LN, Kennedy SH, Parikh SV, et al. The evolution of CANMAT bipolar disorder guidelines: past, present, and future. Bipolar Disord. 2013;15(1):58-60.

8. Taylor DM, Cornelius V, Smith L, Young AH. Comparative efficacy and acceptability of drug treatments for bipolar depression: a multiple-treatments meta-analysis. Acta Psychiatr Scand. 2014;130(6):452-69.

9. Rachid F, Bertschy G, Bondolfi G, Aubry JM. Possible induction of mania or hypomania by atypical antipsychotics: an updated review of reported cases. J Clin Psychiatry. 2004;65(11):1537-45.

10. Khalil RB, Baddoura C. Quetiapine induced hypomania: a case report and a review of the literature. Curr Drug Saf. 2012;7(3):250-3.

11. Pacchiarotti I, Bond DJ, Baldessarini RJ, et al. The International Society for Bipolar Disorders (ISBD) Task Force report on antidepressant use in bipolar disorders. Am J Psychiatry. 2013;170(11):1249-62.

12. Goodwin GM, Haddad PM, Ferrier IN, et al. Evidence-based guidelines for treating bipolar disorder: revised third edition recommendation from the British Association for Psychopharmacology. J Psychopharmacol. 2016;30(6):495-553.

13. Osborne V, Davies M, Layton D, Shakir SA. Utilisation of extended release quetiapine (Seroquel $\mathrm{XL}^{\mathrm{TM}}$ ): results from an observational cohort study in England. Eur Psychiatry. 2016;33:61-7.

14. Silverstone PH, Lalies MD, Hudson AL. Quetiapine and buspirone both elevate cortical levels of noradrenaline and dopamine in vivo, but do not have synergistic effects. Front Psychiatry. 2012;3:82

15. Mundo E, Cattaneo E, Zanoni S, et al. The use of atypical antipsychotics beyond psychoses: efficacy of quetiapine in bipolar disorder. Neuropsychiatr Dis Treat. 2006;2:139-48.

16. Rasmussen H, Ebdrup BH, Aggernaes B, et al. Norquetiapine and depressive symptoms in initially antipsychotic-naive first-episode schizophrenia. J Clin Psychopharmacol. 2013;33:266-9.

17. Sümegi A. Quetiapine in bipolar disorders. Neuropsychopharmacol Hung. 2008;10:281-91.

18. Björkholm C, Jardemark K, Marcus MM, et al. Role of concomitant inhibition of the norepinephrine transporter for the antipsychotic effect of quetiapine. Eur Neuropsychopharmacol. 2013;23:709-20.

19. Altamura AC, Moliterno D, Paletta S, et al. Effect of quetiapine and norquetiapine on anxiety and depression in major psychoses using a pharmacokinetic approach: a prospective observational study. Clin Drug Investig. 2012;32(3):213-9.

20. Rovera C, Mauri MC, Di Pace C, et al. Effect of N-desalkylquetiapine/quetiapine plasma level ratio on anxiety and depression in bipolar disorder: a prospective observational study. Ther Drug Monit. 2017;39(4):441-5. 
21. American Psychiatric Association. Diagnostic and statistical manual of mental disorders, Fifth Revision. Washington, DC: American Psychiatric Press; 2013.

22. Li Q, Su YA, Liu Y, Chen JX, et al. Pharmacokinetics and tolerability of extended-release quetiapine fumarate in Han Chinese patients with schizophrenia. Clin Pharmacokinet. 2014;53(5):455-65.

23. McElroy SL, Martens BE, Creech RS, et al. Randomized, doubleblind, placebo-controlled study of divalproex extended release loading monotherapy in ambulatory bipolar spectrum disorder patients with moderate-to-severe hypomania or mild mania. J Clin Psychiatry. 2010;71(5):557-65.

24. Buoli M, Esposito CM, Godio M, Caldiroli A, Serati M, Altamura AC. Have antipsychotics a different speed of action in the acute treatment of mania? A single-blind comparative study. J Psychopharmacol. 2017:269881117705098. doi:10.1177/ 0269881117705098 (Epub ahead of print).

25. Selle V, Schalkwijk S, Vázquez GH, Baldessarini RJ. Treatments for acute bipolar depression: meta-analyses of placebo-controlled, monotherapy trials of anticonvulsants, lithium and antipsychotics. Pharmacopsychiatry. 2014;47(2):43-52.
26. Ten Have M, Penninx BWJH, Tuithof M, et al. Duration of major and minor depressive episodes and associated risk indicators in a psychiatric epidemiological cohort study of the general population. Acta Psychiatr Scand. 2017;136(3):300-12.

27. Buoli M, Serati M, Altamura AC. Biological aspects and candidate biomarkers for rapid-cycling in bipolar disorder: a systematic review. Psychiatry Res. 2017. doi:10.1016/j.psychres.2017. 08.059 (Epub ahead of print).

28. Hamilton HJ, Gallagher PF, O’Mahony D. Inappropriate prescribing and adverse drug events in older people. BMC Geriatr. 2009;9:5.

29. Nikisch G, Baumann P, Oneda B, et al. Cytochrome P450 and $\mathrm{ABCB} 1$ genetics: association with quetiapine and norquetiapine plasma and cerebrospinal fluid concentrations and with clinical response in patients suffering from schizophrenia: a pilot study. J Psychopharmacol. 2011;25(7):896-907.

30. Buoli M, Dell'Osso B, Caldiroli A, et al. Obesity and obstetric complications are associated with rapid-cycling in Italian patients with bipolar disorder. J Affect Disord. 2017;208:278-83. 